\title{
Study and Review of Various Image Texture Classification Methods
}

\author{
Sandip.S. Patil. \\ Associate Professor \\ Department of Computer Engineering \\ SSBT's College of Engineering \& Technology, \\ Bambhori, Jalgaon, India.
}

\author{
Harshal S. Patil. \\ Research Scholar \\ Department of Computer Engineering \\ SSBT's College of Engineering \& Technology, \\ Bambhori, Jalgaon, India.
}

\begin{abstract}
Pattern is an arrangement of features which are defined by various characteristics of image such as shape, color and texture. Texture is an important characteristic for image analysis. The major trend of the research today in terms of feature extraction for classification is accuracy oriented, however usually the newer algorithms that promises better accuracy is much more complicated in its calculations and often sacrifices the speed of the algorithm.

This paper contains study and review of various techniques used for feature extraction and texture classification. The objective of study is to find technique or combination of techniques to reduce complexity, speed while increasing the accuracy at the same time. Here we are studying and reviewing the three feature extraction methods: Gray Level Co-occurrence Matrix (GLCM), Local Binary Pattern (LBP) and Gabor filter method. Also two classification methods KNN and SVM are used on the texture datasets Brodatz, CUReT, VisTex and OuTex for the experimental purpose.
\end{abstract}

\section{Keywords}

Texture classification, Feature Extraction, Pattern Recognition.

\section{INTRODUCTION}

As humans, it is simple (even for a child) to identify letters, objects, numbers, voices of friends etc. However, to solve these types of problems is a very complex task. Pattern recognition is the science with the objective to classify objects into different categories and classes. It is a primary component of artificial intelligence and computer vision. Pattern recognition methods are used in various areas such as science, engineering, business, medicine and etc.

Texture can be broadly defined as the visual or tactile surface characteristics and appearance of something. Texture is an important characteristic for analysis of many types of images. Texture is present in many real as well as artificial data e.g. clouds, trees, wood, hair, fabric etc. Even though its importance and present everywhere in image data a formal approach or definition of texture analysis does not exist. Texture is a natural property of almost all surfaces the grain of wood, the weave of fabric, the pattern of crop in fields etc. It contains important information about the structural arrangement of surfaces and their relationship to the surrounding environment.

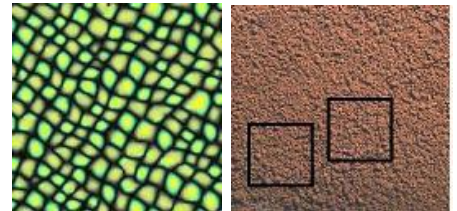

Fig 1: Sample Texture images.

Major issues in texture analysis may be summarized as follows-

1. Given a textured region, classify it in one of a finitely large number of texture classes.

2. To generate a description or model for a class of textured region.

3. Given an image having many distinct textured areas, determine the macro boundaries between different textured regions.

While issue 1 involves the pattern recognition task of texture features estimation, issue 2 deals with identification of generate models of texture. The third important domain performs texture segmentation of an image. Here is the discussion about issue 1 i.e. pattern classification based on texture [1].

The objective of texture classification is to determine the category of a texture image. For example the six small images in Figure 2 can be classified into three categories based on their texture appearance.

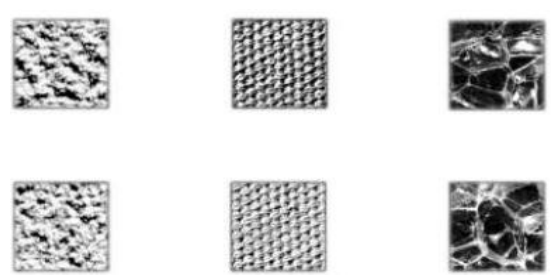

Fig 2: Sample Images for Texture Classification

The usual approach to accomplishing the texture classification task is shown in Figure 3 and texture classification tasks involve two main steps: (1) Feature extraction step, where texture features are extracted from the image and (2) Classification step, where texture class membership is assigned according to the extracted texture features [2]. 


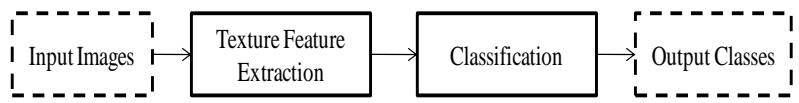

Fig 3: Conventional Texture Classification Procedure.

There are a lot of proposed methods in respect to this field, but most of them were tested using standard image catalogues such as Brodatz and Outex. They might be only well-adjusted and validated for such database, not free for any image database. However, many studies conclude that no single operator is able to recognize every texture database [3].

\section{FEATURE EXTRACTION METHODS}

A large number of feature extraction methods have been proposed and tested in various pattern recognition tasks. There are many different feature extraction methods that were introduced and used for texture classification problems. Most of these methods that were popularly used in recent years were statistical and signal processing methods.

\subsection{Grey Level Co-occurrence Matrices (GLCM)}

Grey Level Co-occurrence Matrices (GLCM) is a statistical method that was proposed by Haralick et.al. back in 1973 which is an important method for feature extraction for texture classification which computes the relationship between pixel pairs in the image. In the textual features were used to be calculated from generated GLCM's like contrast, correlation, energy, entropy etc in conventional methods. Nowadays GLCM method used with other methods and is not used individually. However the major drawback with GLCM method is the computational expense in terms the space required [4].

\section{Flowchart for GLCM:}

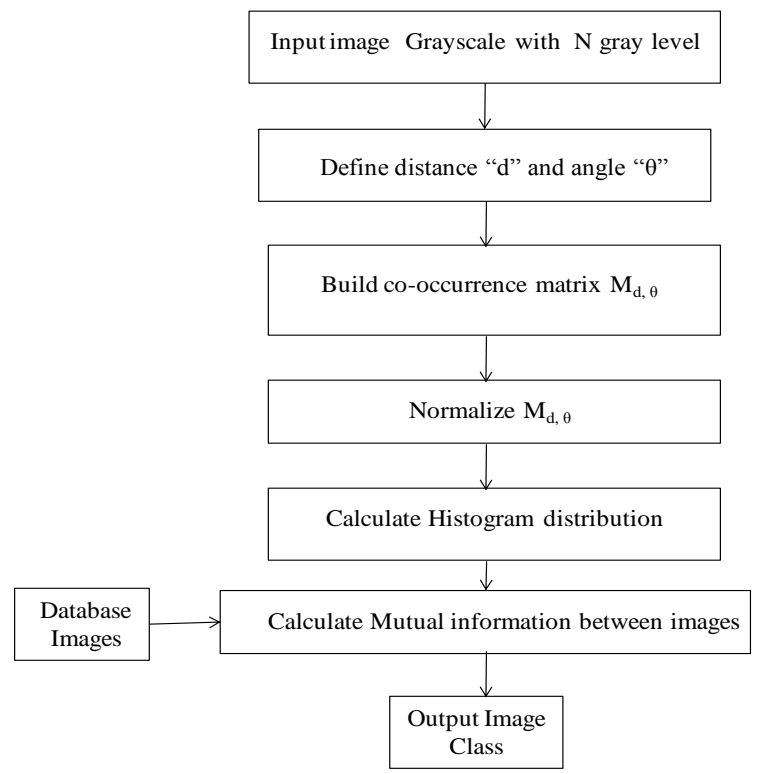

\subsection{Local Binary Pattern (LBP)}

The original Local Binary Pattern (LBP) is proposed by Ojala and Pietikainen back in 1999. It is a Statistical Method. The original LBP calculates a value that reflects the relationship within a $3 \times 3$ neighborhood through a threshold neighborhood that is multiplied with the respective binomial weights. Since the LBP is used to calculate local features, it is often used for texture segmentation problems. It has yet to be a very popular method in texture classification problem [4]. The main drawback of this method is not supportive for scale invariant texture image.

\section{Flowchart for LBP:}
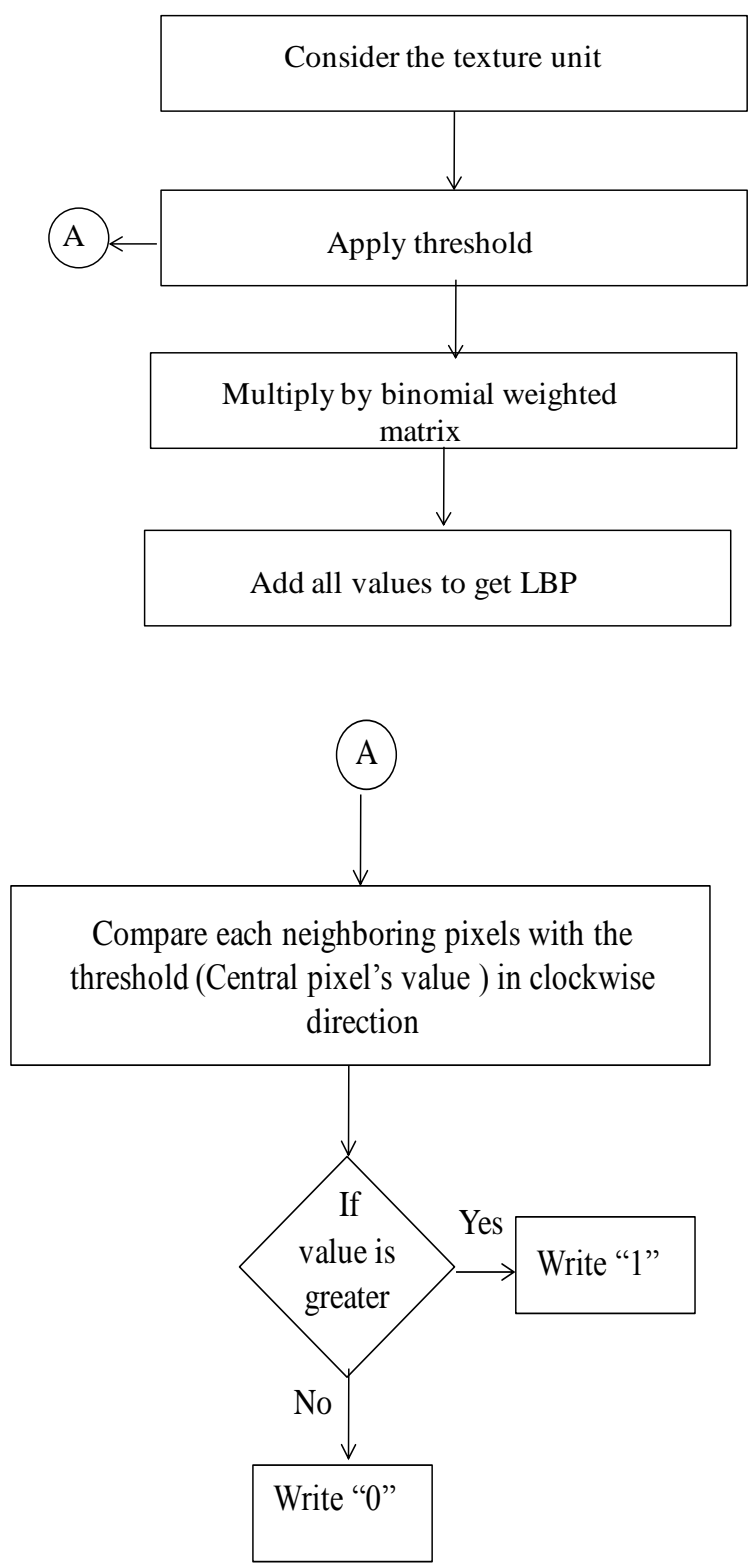

In the database the binomial weighted matrix as shown in figure 4 is saved. The orientation of pixel is also of prime concern. So, the orientation is also described for each pixel as per the mechanism as shown in figure 5 .

Binomial weighted Matrix

\begin{tabular}{|c|c|c|}
\hline 1 & 2 & 4 \\
\hline 8 & - & 16 \\
\hline 32 & 64 & 128 \\
\hline
\end{tabular}

Fig 4: Binomial weighted Matrix 
Texture Units (Pixel Orientation)

\begin{tabular}{|c|c|c|}
\hline Left Top & Top & Right Top \\
\hline Left & Central & Right \\
\hline Left Bottom & Bottom & Right Bottom \\
\hline
\end{tabular}

Fig 5: Pixel orientation texture units

\subsection{Gabor Filters}

Dennis Gabor proposed Gabor features which is a method to represent signals in both the time as well as in frequency domain. These Gabor features are based on Gabor filters (Bovik, 1991; Randen and Husy, 1999), which are used for line detection, finding orientation of an image and also useful for scaling tunable edge. Gabor filters is also known as Gabor wavelets which is a popular signal processing method. The Gabor Filters are defined by parameters: frequency, orientation and standard deviation which cover complete frequency domain by varying these parameters to cover all possible orientations. As the Gabor wavelets methods produces large size, it requires to be downsized. The Principle Component Analysis (PCA) is one of the popular methods to downsize the large space. Gabor filter is still often used in texture classification but sometimes combined with other methods [4, 5].

\section{CLASSIFICATION METHODS}

Texture classification refers to the process of grouping test samples of texture into classes, where each resultant class contains related samples according to some similarity criterion. The goal of classification in general is to select the most appropriate category for an unidentified object, given a set of identified categories. Since perfect classification is repeatedly impossible, the classification may also be performed by determining the probability for each of the known categories.

There are three major groups of classifiers are popularly used, including $k$-Nearest Neighbors, Artificial Neural Networks (ANN) and Support Vector Machines (SVM). Here is discussing $k$-NN and SVM classification methods.

\section{1 k-Nearest Neighbors}

In the method $k$-NN ( $k$ nearest neighbors Fix and Hodges, $1951)$ is a supervised classification method. The $k$-nearest neighbor $(k-\mathrm{NN})$ is an algorithm used in the recognition of patterns for the classification of objects (elements) based on training by examples in the space near the elements. $k$-NN is a type of "Lazy Learning", where function approximates only locally and all computation is delayed to the classification.

Example:

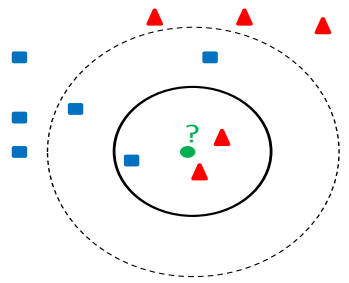

Fig 6: Example of $k$-NN.
The figure 6 shows an example of classification by means $\mathrm{KNN}$. The point under observation is the green dot. The two classes are:

$$
\begin{aligned}
& >\quad \text { that of the red triangles; } \\
& >\quad \text { that the blue squares. }
\end{aligned}
$$

If $k=3$ (i.e. we consider the three nearest objects), then the green dot is placed in the same class of red triangles because there are 2 triangles and 1 square. If $k=5$ is then placed in the same class of blue squares as there are 3 squares and 2 triangles.

In short, the nearest neighbor algorithms are simple classifiers that select the training samples with the closest distance to the question sample. These classifiers will calculate the space from the query sample to every training sample and select the best neighbor or neighbors with the shortest distance.

\section{Flowchart for KNN classifier:}

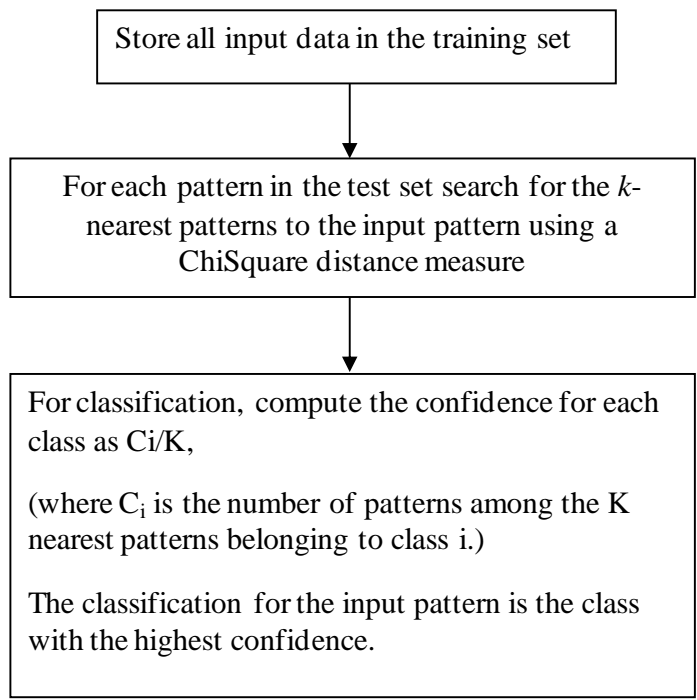

\subsection{Support Vector Machine}

The SVM algorithm was invented by Vladimir N. Vapnik in 1995. SVM is supervised learning classifier. SVM are the newer trends in machine learning algorithm. It is most popular in many pattern recognition problems in current years, with texture classification. SVM is designed to maximize the marginal distance between classes with decision boundaries drawn using different kernels [6]. SVM is designed to work with only two classes by determining the hyperplane to separate two classes. This is done by maximizing the margin from the hyperplane to the two classes. The samples closest to the margin that were selected to determine the hyperplane is known as support vectors. Multiclass classification is also applicable, the multiclass SVM is basically built up by various two class SVMs to solve the problem, either by using one versus all or one versus one. The winning class is then determined by the highest output function or the maximum votes respectively. Despite that, SVM is still considered to be powerful classifier which was replacing the ANN and has slowly evolved into one of the most important main stream classifier. They are now widely used in the research of texture classification. 


\section{TEXTURE DATASETS}

There are a number of texture databases or datasets that were used in experiments on texture classification and which are freely available for comparison of texture analysis algorithms, e.g. the Brodatz texture album, OuTex dataset and CUReT texture dataset which were more widely used.

\subsection{Brodatz Texture Album}

The oldest database is Brodatz texture database prepared by Phil Brodatz in 1966, which contain grey-scale images and it is freely available for research purposes at (database Brodatz). The Brodatz textures are popular and widely used as standard datasets in texture classification. It is consists of 112 textures from the Brodatz texture album [7].

Each of these textures is produced from a single image scanned from the texture album. Although the Brodatz textures are widely used, there are many different subsets of the dataset which often involves only part of the album and some with rotated and scaled samples added. The entire dataset are sometimes also used [8]. Sample images of the Brodatz textures are shown in Figure 7.

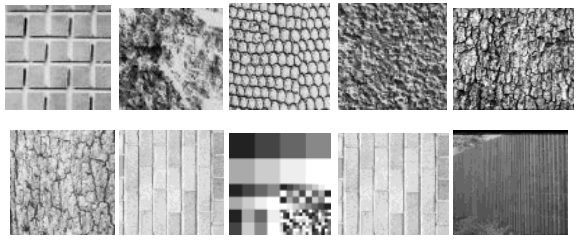

Fig 7: Samples of four images from the Brodatz texture album.

The main drawback of the Brodatz texture dataset is that all the textures are represented by a single image only. Therefore, the users need to segment to dataset into smaller segments, and frequently scale and rotate them. Because there is only a single image for each texture, the segments of the same texture will be slightly homogeneous for both training and testing samples $[4,9,10]$.

\subsection{CUReT Dataset}

The Columbia-Utrecht Reflectance and Texture (CUReT) by Dana et al.(1999), dataset used for recognition tests, the dataset can be downloaded from (dataset CUReT). The CUReT dataset is produced in a collaborated research between Columbia University and Utrecht University [11]. The Columbia-Utrecht (CUReT) database contains 61 different textures with 92 images with each class that we commonly see in our surroundings. It contains rough textures, those which have specularities, exhibit anisotropy, are manmade and many others. It contains the images under 205 different rotation and illumination conditions. This dataset doesn't contain the scale invariant images. Multiple instances of same texture are present for only a very few of the materials. The variety of textures present in the database is shown in Figure 8.

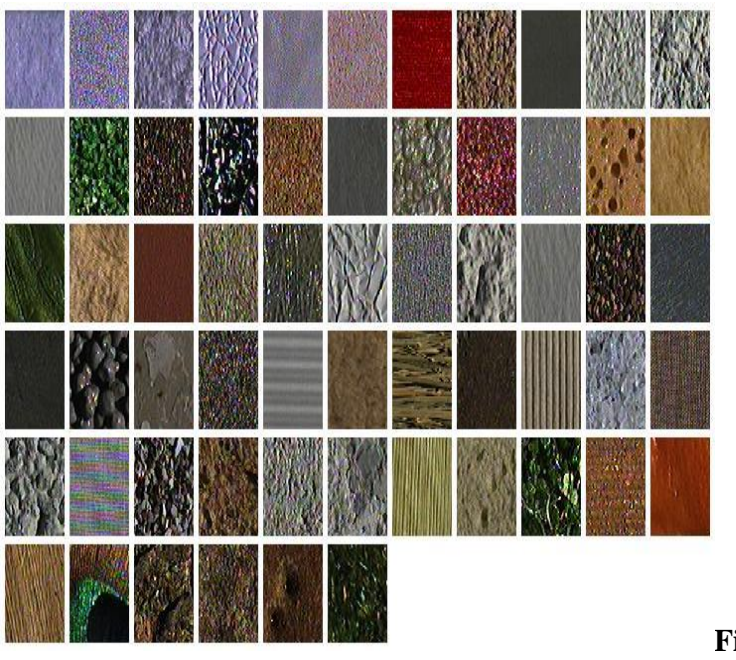

8: One image of each of the textures present in the Columbia-Utrecht database.

\subsection{VisTex Dataset}

The Vision Texture (VisTex) dataset is prepared by the Massachusetts Institute of Technology (MIT) [12]. The dataset is not only consisting of homogeneous frontal acquisition of textures, it also comes with real-world scenes with multiple textures and video textures.

The database was created with the intention of providing a large set of high quality textures for computer vision applications. In particular, the set was made as an choice to the Brodatz texture collection, Brodatz album is not freely available for research use. The goal of VisTex is to provide texture images that are representative of real world conditions. VisTex database contains color texture images. Sample images of the dataset are shown in Figure 9.
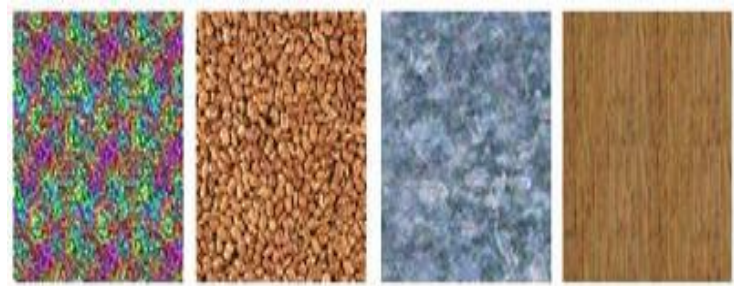

Fig 9: Examples of four textures from the VisTex dataset.

\subsection{OuTex Dataset}

The OuTex dataset include variations of illumination, rotation or both of them. The best variations of illumination spectra is comprised in Outex database (Ojala et al., 2002a), which consists of color texture images acquired under three different illumination spectra and nine in-plane rotations. Outex also define several classification tests, which differ in recognition conditions.

The collection of surface textures is expanding continuously. The database contains 320 surface textures, both macrotextures and microtextures. It is also most popular and largest dataset. [13, 14]. Sample images of the OuTex textures are shown in Figure 10. 


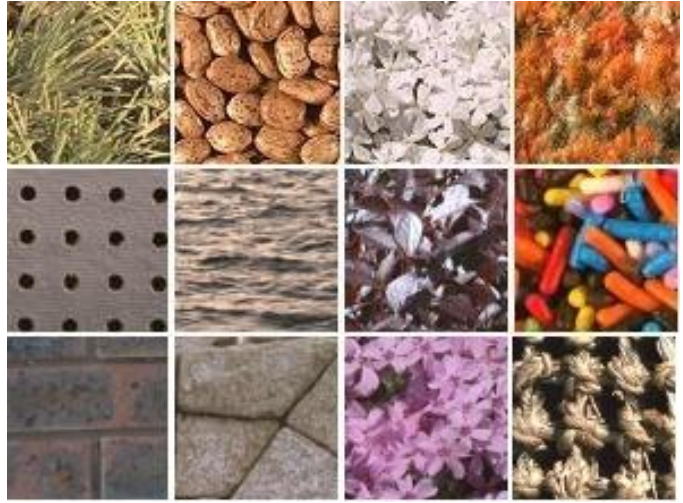

Fig 10: Images of OuTex database

The table shows overview of various efforts taken by the researchers in the field of image texture classification. Accuracy and approach are of prime concern for this overview.

Table 1: Review of Image Texture Classification Techniques

\begin{tabular}{|c|c|c|c|c|c|}
\hline $\begin{array}{l}\text { Sr. } \\
\text { No. }\end{array}$ & Authors, year & Approach & \multicolumn{2}{|c|}{ Technique } & $\begin{array}{c}\text { Accuracy } \\
(\%)\end{array}$ \\
\hline \multirow[t]{2}{*}{1} & \multirow{2}{*}{$\begin{array}{c}\text { Tou et al, } 2009 \\
\text { [17] }\end{array}$} & \multirow{2}{*}{$\begin{array}{c}\text { Co-occurence } \\
\text { matrix }\end{array}$} & \multicolumn{2}{|c|}{ GLCM (12 features) } & 85.73 \\
\hline & & & \multicolumn{2}{|c|}{ GLCM (10 features) } & 78.15 \\
\hline \multirow[t]{3}{*}{2} & \multirow{3}{*}{$\begin{array}{l}\text { Tuzel et al, } \\
2006[18]\end{array}$} & \multirow{3}{*}{$\begin{array}{l}\text { Covariance } \\
\text { matrix }\end{array}$} & \multicolumn{2}{|c|}{ Edge base derivatives } & 84.65 \\
\hline & & & \multicolumn{2}{|c|}{ GLCM } & 79.94 \\
\hline & & & \multicolumn{2}{|c|}{ Gabor filter } & 91.86 \\
\hline \multirow[t]{2}{*}{3} & \multirow{2}{*}{$\begin{array}{c}\text { Tou et al, } 2009 \\
\text { [17] }\end{array}$} & \multirow[t]{2}{*}{ Filters } & \multicolumn{2}{|c|}{ Gabor 12 filters } & 89.74 \\
\hline & & & \multicolumn{2}{|c|}{ Gabor 24 filters } & 91.86 \\
\hline \multirow[t]{4}{*}{4} & \multirow{4}{*}{$\begin{array}{c}\text { Zhao et al, } 2011 \\
{[16]}\end{array}$} & \multirow{4}{*}{$\begin{array}{l}\text { Structural and } \\
\text { statistical }\end{array}$} & \multirow[t]{2}{*}{ LBP } & Broadatz & 79.97 \\
\hline & & & & UIUC & 57.59 \\
\hline & & & \multirow[t]{2}{*}{ CLBP } & Broadatz & 86.63 \\
\hline & & & & UIUC & 83.29 \\
\hline 5 & $\begin{array}{c}\text { Tou et al, } 2009 \\
\text { [17] }\end{array}$ & Combination & \multicolumn{2}{|c|}{ GLCM and Gabor } & 91.06 \\
\hline
\end{tabular}

It is easily noticeable that signal processing methods are very popularly used in the recent years, especially for Gabor filters. Although this method required more computation as they are examining the frequency domain, the accuracy obtained is good and usually outperform older and simpler techniques. The old technique like GLCM is however yet to be forgotten in the field of texture classification because it is one of the simplest textural feature which is old but is computationally inexpensive. The LBP method has been widely used in texture analysis because it is excellent property of gray scale invariant also it is theoretically simple but very powerful. The major trend of the research today in terms of feature extraction for texture classification is accuracy oriented, however usually the newer algorithms that promises better accuracy is much more complicated in its calculations and often sacrifices the speed of the algorithm.

SVM is today not only the major trend in texture classification, it is also usually a very trendy classifier in various pattern recognition problems, including recognition and detection problems. However it was not initially designed for multiclass problems, therefore it is adapted to implement of multiclass problem which is more complicated and will be slightly slower. In the $k$-nearest neighbors all sample points have to be stored but in SVM only the chosen samples which are of good representation to classify the problem space will be stored and known as support vectors.

The common problem of texture classification today is that texture datasets that are available in the field are usually having each textures acquired only once, therefore each class often have very homogeneous training and testing samples. This problem has not been solved due to the difficulty in preparing datasets with different acquisition of a same type of texture for all the textures. The Brodatz texture album is a printed album that is available backed in 1996. Other newer datasets have yet to be very popularly used but has often included more aspect, such as variations in viewing angle and illumination. The OuTex dataset is popular for variations of illumination, direction or both of them.

The table 1 shows review of various efforts taken by the researchers in the field of image texture classification. Accuracy and approach are of prime concern for this overview. Accuracy of $85.73 \%$ is achieved using the cooccurrence matrix approach for 12 features is achieved by Tou et al., The same paper describes $91.86 \%$ accuracy is provided by Gabor filter technique. The accuracy is directly proportional to number of filters (for 12 Gabor filter accuracy is $89.74 \%$ and for 24 Gabor filter accuracy is $91.86 \%$ ). However the combination of GLCM and Gabor filter provide $91.06 \%$ accuracy. Edge based derivative technique proposed by Tuzel et al, provides $84.65 \%$ accuracy, which is implemented using co-variance matrix. Complete Local binary pattern provides better performance over LBP approach for database like Broadatz and UIUC. This structural and statistical approach is described by Zhao et al. The table 1 shows that by using Gabor filter technique and the combination of both Gabor filter and GLCM technique gives better result for image texture classification.

\section{CONCLUSION}

The choice of Gabor filter and GLCM gives highest accuracy as compare to other methods like LBP if the complexity of algorithms is higher. For classification of featured image $k$ $\mathrm{NN}$ is most suitable classifier on the account of its faster speed.

In future it is plan to improve accuracy and speed of both feature extraction and texture classification by implementing LBP method for feature extraction and for classification will use two classifiers first is SVM and second is $k$-NN which is comparatively simple algorithm. And Outex dataset will be used to verify the results.

\section{REFERENCES}

[1] R.J.Bhiwani, S.M.Agrawal and M.A.Khan,2010. "Texture Based Pattern Classification", International Journal of Computer Applications, Vol. 1 - No. 1, pp. 60-62.

[2] Andy song,2003. "Texture Classification: A Genetic Programming Approch", April 9,

[3] Prasetiyo, Marzuki Khalid, Rubiyah Yusofand Fabrice Meriaudeau,2010. "A Comparative Study of Feature Extraction Methods for Wood Texture Classification", Sixth International Conference on Signal-Image 
Technology and Internet Based Systems, vol. 1, pp. 2329.

[4] Jing Yi Tou, Yong Haur Tay and Phooi Yee Lau,2009. "Recent Trends in Texture Classification: A Review" Symposium on Progress in Information \& Communication Technology, pp. 63-68.

[5] J.Y. Tou, Y.H. Tay, and P.Y. Lau,2009. "Gabor Filters as Feature Images for Covariance Marix on Texture Classification Problem," ICONIP 2008, vol. 5507, pp. 745-751.

[6] C. Chen, C. Chen and C. Chen, 2006. "A Comparison of Texture Features Based on SVM and SOM," ICPR, vol. 2, pp. 630-633.

[7] P. Brodatz, 1996. Textures: A Photographic Album for Artists and Designers, Dover, New York.

[8] Scott Blunsden, 2004. "Texture Classification using Non-Parametric Markov Random Fields" MSUI University of Edinburgh.

[9] Information about Brodatz texture database: http://www.ux.uis.no/ tranden/brodatz.html

[10] Theory regarding Brodatz texture database: http://www.texturesynthesis.com/meastex/imgs/brodatz.h tml

[11] Information about CUReT texture dataset: http://www1.cs.columbia.edu/CAVE/software/curet

[12] The Vision Texture (VisTex) dataset is prepared by the Massachusetts Institute of Technology: http://vismod.media.mit.edu/vismod/imagery/VisionText ure/

[13] Information about outex texture dataset: http://www.outex.oulu.fi/temp/orig.html

[14] S. Liao, M.W.K. Law, A.C.S. Chung, 2009. "Dominant Local Binary Patterns for Texture Classification," IEEE $T I P$, vol. 18 , no. 5 , pp. 1107-1118.
[15] Zhao et al, 2011. "Texture Classification Based on Completed Modelling of Local Binary Pattern" IEEE International Conference on Computational and Information Science, vol. 2, pp. 268-271.

[16] J. Y. Tou, Y. H. Tay and P.Y. Lau, 2009. "A Comparative Study for Texture Classification Techniques on Wood Species Recognition Problem" IEEE Fifth International Conference on Natural Computation, pp. 8-12.

[17] O. Tuzel, F. Porikli and P. Meer, 2006. "Region Covariance: A Fast Desscriptor for Detection and Classification", European Conference on Computer Vision, vol. 1, pp. 697-704.

\section{About the Authors}

Sandip S. Patil received the B.E. degree in Computer Engineering, in 2001 from North Maharashtra University Jalgaon (M.S.), M.Tech. in Computer Science and Engineering from Samrat Ashok Technological Institute Vidisha in 2009, Presently working as Associate Professor in department of Computer Engineering at S.S.B.T. College of Engineering and Technology, Bambhori, Jalgaon. (India), having 12 years of research experience. His area of interests is Pattern Recognition, machine learning and Soft Computing. He achieved Promising Engineer Award-2011 and Young Engineer Award-2013 of I.E. India.

Harshal S. Patil received the B.E. degree in Computer Engineering, in 2010 from North Maharashtra University, Jalgaon (M.S.). She is presently pursuing M.E.in Computer Science and Engineering from North Maharashtra University, Jalgaon (M.S.). Her area of interests is Pattern Recognition and Image Processing. 